\section{Common Kibra Alleles Are Associated with Human Memory Performance}

\author{
Andreas Papassotiropoulos, ${ }^{1,3 *}$ Dietrich A. Stephan, ${ }^{3 *}+$ Matthew ]. Huentelman, ${ }^{3}$ \\ Frederic J. Hoerndli, ${ }^{1}$ David W. Craig, ${ }^{3}$ John V. Pearson, ${ }^{3}$ Kim-Dung Huynh, ${ }^{1}$ \\ Fabienne Brunner, ${ }^{1}$ Jason Corneveaux, ${ }^{3}$ David Osborne, ${ }^{4}$ M. Axel Wollmer, ${ }^{1}$ Amanda Aerni, ${ }^{1}$ \\ Daniel Coluccia, ${ }^{1}$ ]ürgen Hänggi, ${ }^{1}$ Christian R. A. Mondadori, ${ }^{1}$ Andreas Buchmann, ${ }^{1}$ \\ Eric M. Reiman, ${ }^{3,6}$ Richard ]. Caselli, ${ }^{5}$ Katharina Henke, ${ }^{1}$ Dominique J.-F. de Quervain ${ }^{1,2}$
}

Human memory is a polygenic trait. We performed a genome-wide screen to identify memoryrelated gene variants. A genomic locus encoding the brain protein KIBRA was significantly associated with memory performance in three independent, cognitively normal cohorts from Switzerland and the United States. Gene expression studies showed that KIBRA was expressed in memory-related brain structures. Functional magnetic resonance imaging detected KIBRA alleledependent differences in hippocampal activations during memory retrieval. Evidence from these experiments suggests a role for KIBRA in human memory.

$\mathrm{H}$ uman memory is a polygenic cognitive trait. Heritability estimates of $\sim 50 \%$ suggest that naturally occurring genetic variability has an important impact on this fundamental brain function (1). Recent candidategene association studies have identified some genetic variations with significant impact on human memory capacity (2-5). However, the success of these studies depends upon preexisting information, which limits their potential to identify unrecognized genes and molecular pathways $(6,7)$.

Recent advances in the development of high-density genotyping platforms now allow for high-resolution whole-genome association studies for polygenic phenotypes $(8,9)$. We used a genome-wide screen with more than 500,000 single-nucleotide polymorphisms (SNPs). The initial screen was done in pooled DNA from a Swiss cohort that was stratified into groups of participants with different levels of episodic memory performance (verbal delayed recall). SNPs fulfilling the selection criteria of the pooling stage (10) were genotyped individually in this same cohort and validated in two additional cohorts from the United States and from Switzerland.

We recruited 351 young adults (median age, 22 years; range, 18 to 48 years) from Switzerland (Swiss cohort 1) (10). Genetic association studies in outbred populations such as this one may be prone to false positives because nonrandom genetic heter-

${ }^{1}$ Division of Psychiatry Research, ${ }^{2}$ Center for Integrative Human Physiology, University of Zurich, Zurich 8057, Switzerland. ${ }^{3}$ Neurogenomics Division, Translational Genomics Research Institute, Phoenix, AZ 85004, USA. ${ }^{4}$ Department of Psychology, ${ }^{5}$ Department of Neurology, Mayo Clinic, Scottsdale, AZ 85259, USA. ${ }^{6}$ The Banner Alzheimer's Institute and the Department of Psychiatry, University of Arizona, Phoenix, AZ 85006, USA.

*These authors contributed equally to this work. †To whom correspondence should be addressed. E-mail: papas@bli.unizh.ch (A.P.); dstephan@tgen.org (D.A.S.) ogeneity within the study sample (population structure) can lead to spurious associations between a genetic marker and a phenotype (11). Therefore, we controlled for genetic background and found no evidence of significant population stratification; the participants' genetic backgrounds formed one normally distributed cluster $(P=0.6)(10,12)$. We identified 10 participants as outliers (probability of cluster allocation lower than $25 \%$ ) and excluded them from the genetic association studies. The remaining population $(n=$ 341) was stratified into four groups according to their performance in a verbal memory task which quantified the retrieval success $5 \mathrm{~min}$ after learning a word list comprising 30 semantically unrelated nouns (10). Each of these groups was genotyped at 502,627 SNPs. To minimize the possibility of false positives (at the apparent cost of false negatives), we combined two different and stringent statistical approaches (a single-point method and, as a filter for the most significant SNPs, a sliding-window method) to select SNPs of

Table 1. Association of SNPs rs17070145 (KIBRA) and rs6439886 (CLSTN2) with verbal episodic memory in Swiss cohort 1. Genotype calls of eight subjects were not considered for analysis due to low-quality pyrograms for rs17070145. Means with common superscripts are significantly different according to multifactorial analysis of variance. Data are means \pm SEM.

\begin{tabular}{|c|c|c|c|c|}
\hline & \multicolumn{4}{|c|}{ No. of words recalled } \\
\hline & $n$ & Immediately & $\begin{array}{l}\text { After } \\
5 \text { min }\end{array}$ & $\begin{array}{c}\text { After } \\
24 \text { hours }\end{array}$ \\
\hline \multicolumn{5}{|c|}{ rs17070145 } \\
\hline CC & 164 & $23.6 \pm 0.3$ & $7.6 \pm 0.2^{*}$ & $6.7 \pm 0.2 \dagger$ \\
\hline $\mathrm{CT} / \mathrm{TT}$ & 169 & $24.1 \pm 0.3$ & $9.4 \pm 0.2^{*}$ & $8.0 \pm 0.2 \dagger$ \\
\hline \multicolumn{5}{|c|}{ rs6439886 } \\
\hline$\pi$ & 265 & $23.9 \pm 0.2$ & $8.4 \pm 0.2 \ddagger$ & $7.3 \pm 0.2 \S$ \\
\hline $\mathrm{TC} / \mathrm{CC}$ & 76 & $24.2 \pm 0.4$ & $9.8 \pm 0.4 \ddagger$ & $8.4 \pm 0.4 \S$ \\
\hline
\end{tabular}

high statistical confidence (10). Two SNPs fulfilled these selection criteria and were prioritized for subsequent individual genotyping to exclude pooling-related false positives: rs17070145 and rs6439886. Both SNPs map within genes expressed in the human brain: rs17070145 is a common $\mathrm{T} \rightarrow \mathrm{C}$ substitution within the ninth intron of KIBRA (GenBank accession number NM_015238), encoding a neuronal protein, and rs6439886 is a common $\mathrm{T} \rightarrow \mathrm{C}$ substitution within the first intron of CLSTN2 (encoding the synaptic protein calsyntenin 2) (NM_022131).

Both the KIBRA and CLSTN2 SNPs were also significantly associated with differential human memory performance when we genotyped them individually in Swiss cohort 1 using an independent genotyping technology (10). Carriers of KIBRA rs $17070145 \mathrm{~T}$ allele had $24 \%$ better free recall performance 5 min after word presentation $(P=0.000004)$ and $19 \%$ better free recall performance 24 hours after word presentation $(P=0.0008)$ than did noncarriers (Table 1 , table $\mathrm{S} 1$, and fig. S2). TT and CT genotype groups of rs17070145 were combined because the frequency of the TT genotype was low and because both groups displayed similar memory performance (table S1). SNP rs6439886 yielded similar results; however, the mean difference of memory performance between genotype groups was lower than that of rs17070145 (Table 1 and table S1). Both the 5-min and the 24-hour delayed free recall reflected episodic, hippocampus-dependent memory (13). Neither SNP was associated with performance on immediate recall tests (Table 1 and table S1), indicating that the alleledependent differences in episodic memory were not caused by allelic effects on con-

Table 2. Association of SNPs rs 17070145 (KIBRA) and rs6439886 (CLSTN2) with episodic memory in the U.S. population. The SRT was completed by 200 participants ( 98 CC carriers and 102 CT and $\Pi$ carriers of rs17070145). Genotype calls of seven participants were not considered for analysis because of low-quality pyrograms for rs6439886. Means with common superscripts are significantly different according to multifactorial analysis of variance. Data are means \pm SEM.

No. of items recalled

\begin{tabular}{|c|c|}
\hline $\begin{array}{r}\text { mmed } \\
\text { (AV }\end{array}$ & $\begin{array}{c}\text { After } \\
30 \mathrm{~min} \\
\text { (AVLT) }\end{array}$ \\
\hline
\end{tabular}

\begin{tabular}{lrrrr} 
rs 17070145 \\
CC & 126 & $9.4 \pm 0.3$ & $8.5 \pm 0.3 *$ & $83.7 \pm 1.2 \dagger$ \\
CT/TT & 130 & $10.0 \pm 0.3$ & $9.7 \pm 0.3 *$ & $90.3 \pm 1.1 \dagger$ \\
rs6439886 & & & \\
$\pi$ & 185 & $9.7 \pm 0.2$ & $9.1 \pm 0.2$ & $88.4 \pm 0.9$ \\
$T C / C C$ & 64 & $9.9 \pm 0.4$ & $9.2 \pm 0.4$ & $88.9 \pm 1.6$ \\
\hline${ }^{*}=0.004$ & $\dagger P=0.00005$
\end{tabular}


founding factors such as motivation, attention, or working memory.

Both SNPs were further evaluated in a second, independent population of $256 \mathrm{cog}$ nitively normal participants (median age, 55 years; range, 20 to 81 years) from the United

Table 3. Association of SNP rs17070145 (KIBRA) with visual episodic memory in Swiss cohort 2. Data are means \pm SEM.

\begin{tabular}{lcccc} 
& $n$ & $\begin{array}{c}\text { Pictures } \\
\text { recalled after } \\
\text { 10 } \text { min }\end{array}$ & $\begin{array}{c}\text { Attention } \\
\text { (correct } \\
\text { characters) }\end{array}$ & $\begin{array}{c}\text { Digit span } \\
\text { (correct } \\
\text { series) }\end{array}$ \\
\hline $\mathrm{CC}$ & 191 & $4.5 \pm 0.1^{*}$ & $157 \pm 2$ & $8.9 \pm 0.2$ \\
$\mathrm{CT} / \mathrm{TT}$ & 233 & $5.0 \pm 0.1^{*}$ & $158 \pm 2$ & $8.8 \pm 0.2$ \\
\hline
\end{tabular}

Fig. 1. Significance of SNPs and haplotypes in Swiss cohort 1. Individual genotyping in Swiss cohort 1 confirmed poolingbased screening results and implicated the KIBRA locus in memory performance. Black dots indicate the significance level of SNPs. Continuous red horizontal lines represent the significance level of specific haplotypes. Chromosome position in base pairs from the $p$ terminus of chromosome 5 for each SNP is given on the $x$ axis. Intronic regions of the ODZ2, KIBRA, and RARS genes are given in shaded gray, and short vertical lines represent the exons. The lower panel visualizes the haplotype structure of the examined region as assessed by Haploview 3.2 (29). Red squares represent regions of high degree of LD $\left(D^{\prime}>0.8\right)$. Blue squares represent regions of high LD but for likelihood of odds (LOD) ratio.
States. The KIBRA SNP showed significant association with episodic memory with the same direction of effect: $\mathrm{T}$ allele carriers had significantly better memory scores than noncarriers in the Buschke's Selective Reminding Test (SRT) (14) (Table 2). Performance on another episodic memory task, the Rey Auditory Verbal Learning Test (AVLT) (15), was also significantly different between allele groups (Table 2). There were no alleledependent differences in the outcome of the Wisconsin Card Sorting Test or on the Paced Auditory Serial Attention Task, suggesting that rs 17070145 was not associated with executive functions, attention, or working memory in this population. As expected, the U.S. cohort displayed substantial allelic divergence. All but 18 participants reported that they were of non-Hispanic European ancestry; the 18 exceptions were of Hispanic (9), African-American (4), Native-American (3), and Asian (2) ancestry. Exclusion of these 18 individuals had no effect on the allelic association with memory performance in this sample. Genomic control analysis showed that the exclusion of these 18 participants reduced the inflation factor $\lambda$ from 1.6 to 1.2 , which still indicates the presence of notable genetic heterogeneity. However, inclusion of an individual's genetic background value as a covariate had no influence on the association of the KIBRA genotype with episodic memory performance in this sample (10). CLSTN2 SNP rs6439886 failed to show significant association with episodic memory in this older population
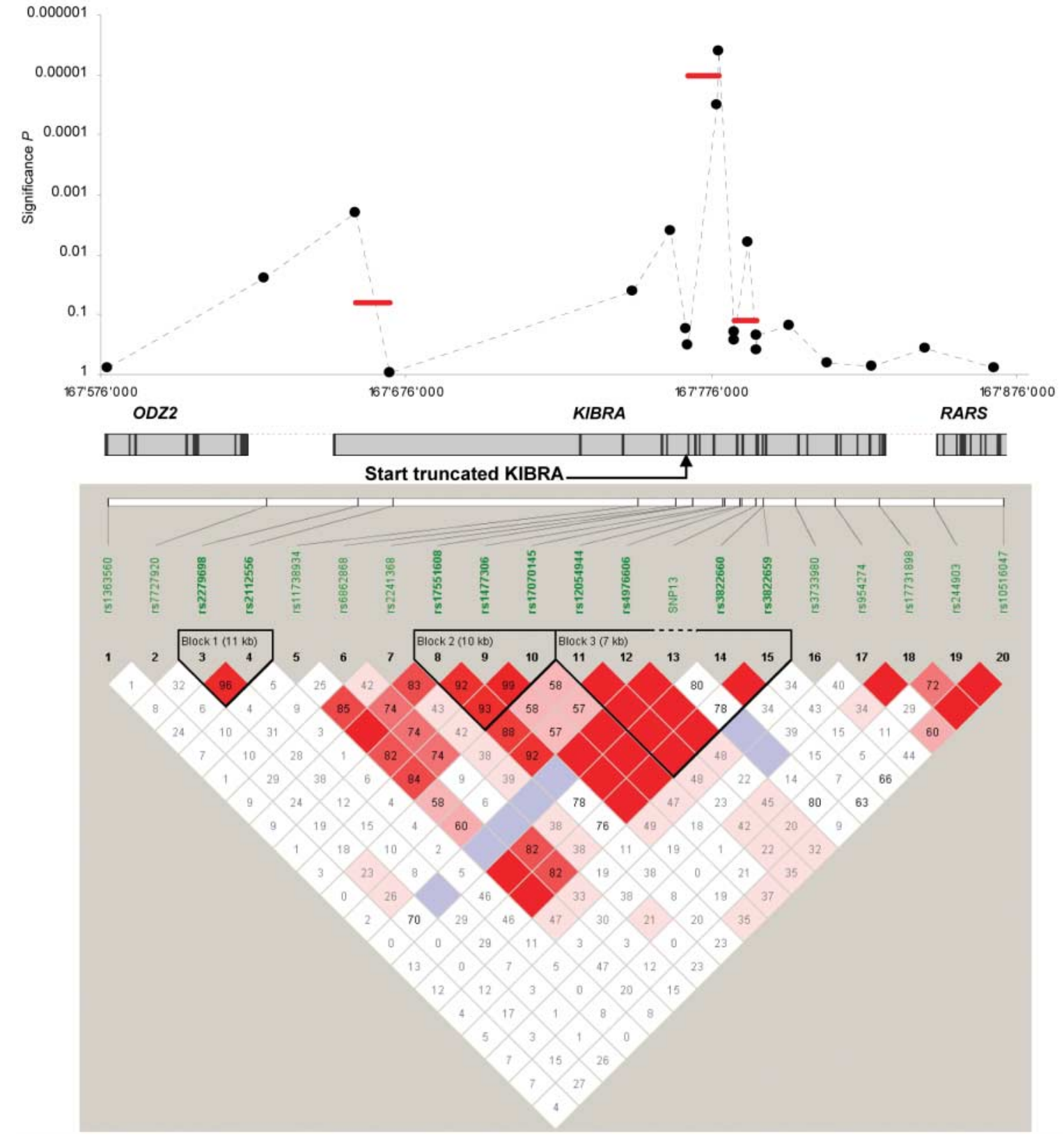
(Table 2). The lack of significance in the U.S. population for that particular SNP may be related to differences in ethnicity or to differences in mean age between the populations.

KIBRA SNP rs17070145 was further evaluated in a third population of 424 young adults (median age, 21 years; range, 18 to 28 years) from Switzerland (Swiss cohort 2), who performed a visual episodic memory task (10). Structured association and genomic control analysis with 122 SNPs revealed a genetically homogeneous cohort $(\lambda=1.008)$. $\mathrm{T}$ allele carriers also performed significantly better than did noncarriers in this population (Table 3). There were no allele-dependent differences in the outcomes of the control tasks (i.e., d 2 cancellation test and the digitspan task), suggesting that rs 17070145 was also not associated with attention, concentration, or working memory in this population. Taken together, we found evidence for association of the $\mathrm{T}$ allele of rs 17070145 with better episodic memory performance in three independent study populations, whereas no significant associations were found with performance in cognitive control tasks of attention, concentration, and working memory (table S2). The allelic distribution of rs17070145 differs significantly between eth- nic groups according to the National Center for Biotechnology Information database of genetic variation (dbSNP). In populations of European ancestry, the $\mathrm{T}$ allele is the minor one with a frequency of $\sim 25 \%$, as also shown in this study. In contrast, in Asian populations the $\mathrm{T}$ allele is most frequent $(75 \%)$ and in African-American populations, the $\mathrm{T}$ and $\mathrm{C}$ alleles are almost equally frequent (54\% and $46 \%$, respectively). Therefore, it would be interesting for subsequent studies to assess $K I B R A$ 's relation to memory in populations of non-European ancestry.

Fine-mapping the genomic region harboring KIBRA and the flanking genes RARS and $O D Z 2$ with 19 additional SNPs in Swiss cohort 1 (Fig. 1) was performed to ensure that the observed association of KIBRA SNP rs17070145 with episodic memory was not due to linkage disequilibrium (LD) with genetic variations in nearby genes. Three haplotype blocks were observed within KIBRA with this set of SNPs (Fig. 1). SNP rs17070145 and the corresponding haplotype block (block 2) yielded the highest significance levels. We concluded that the observed association is unrelated to LD with adjacent genes (Fig. 1). Further fine-mapping of 32 kilobases encompassing KIBRA haplotype block 2 with 58

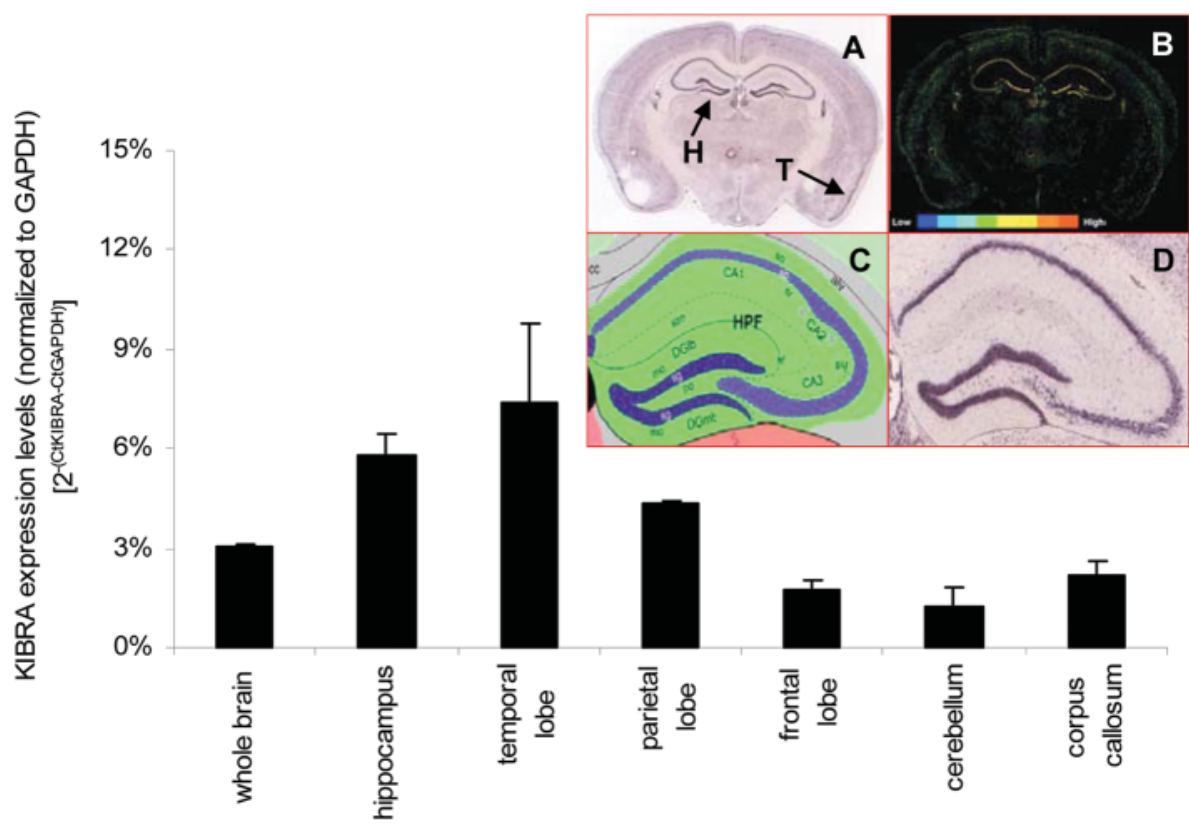

Fig. 2. KIBRA expression in memory-related structures in human and murine brain. Expression levels of truncated KIBRA in humans were measured by quantitative RT-PCR with data normalized to glyceraldehyde-3-phosphate dehydrogenase (GAPDH) expression levels. Error bars indicate SD. The inset shows the expression of KIBRA in the hippocampus and the temporal lobe of the murine brain as detected by in situ hybridization (30). Dark purple staining regions in (A) and (D) indicate areas of high KIBRA expression. (B) A gene expression filter with yellow and red color indicates areas of high gene expression. (C) An illustration of the relative atlas region. $\mathrm{H}$, hippocampus; $\mathrm{T}$, temporal cortex. Panels in the inset show sections scanned at high resolution $(10 \times$ magnification, $0.95 \mu \mathrm{m} / \mathrm{pixel})$. Panel (A) shows a coronal section of the murine brain at coronal level 72 , and panel (D) is a $5 \times$ magnification of $(A)$ showing the hippocampal formation. Data provided under license by the Allen Institute for Brain Science.

additional SNPs refined the haplotype structure of this region and revealed the existence of additional SNPs with significance levels comparable to those of rs17070145 (fig. S1). This can be fully attributed to the tight LD between these SNPs. As part of our quality control, the most significant KIBRA SNPs were genotyped twice in two independent laboratories with different singleplex genotyping technologies (Pyrosequencing and Amplifluor). Genotyping scoring was done manually and in a blind manner, and the level of congruence was $>99 \%$. Because missing genotype calls may inflate test statistics (16), we investigated the influence of calling failures on the association results of Swiss cohort 1 . In 205 individually genotyped SNPs, the average calling failure rate was $3.9 \%$ for SNP assays and $2.4 \%$ for study subjects. Neither value correlated significantly with memory performance $(P>0.5)$. Inclusion of calling failures as covariate had no influence on the association of KIBRA SNPs with memory performance.

We determined expression levels of $K I B R A$ in memory-related human brain regions with reverse transcription polymerase chain reaction (RT-PCR) amplicons designed to detect both KIBRA full-length transcript and its truncated form KIAA0869, which lacks the first 223 amino acids (17). We also studied the protein expression of truncated KIBRA with immunocytochemistry and Western blotting in fresh frozen brain tissue (temporal lobe) from 14 individuals (fig. S5). RNA expression levels of fulllength KIBRA in the human brain were almost undetectable. In contrast, expression levels of truncated KIBRA were high in all memory-related structures in the human brain, including the hippocampus and the temporal lobe (Fig. 2). Furthermore, in situ hybridization studies in mice showed that KIBRA expression is highest in the dentate gyrus and the CA1 region of the hippocampal formation (Fig. 2), two key regions for memory.

Functional magnetic resonance imaging (fMRI) was done to study the relation of the KIBRA genotype to human memory-related neuronal activity. Thirty participants from Swiss population 1 (15 carriers of the rs17070145 T allele versus 15 noncarriers) underwent fMRI. The allelic groups were matched for sex (5 males and 10 females in each group), education $(P=0.7)$, age $(P=0.8)$, genetic heterogeneity $(P=0.5)$, and for two polymorphisms previously shown to be related to memory performance - that is, the $\mathrm{His}^{452} \rightarrow \mathrm{Tyr}^{452} \mathrm{SNP}$ of the HTR $2 A$ gene $(P=0.4)$ and the $\mathrm{Val}^{66} \rightarrow \mathrm{Met}^{66} \mathrm{SNP}$ of the $B D N F$ gene $(P=$ $0.5)$. Furthermore, the groups were matched for 5-min delayed recall performance $(P=1)$ to avoid measuring genotype-unrelated performance effects on brain activations and to 
instead capture genotype-dependent differences in brain-activation patterns. We expected that noncarriers of the $\mathrm{T}$ allele would need more activation in memory-related brain regions to reach the same level of memory performance (18). KIBRA has been associated with human episodic memory, which depends on the function of the hippocampus $(13,19,20)$, leading us to also hypothesize that KIBRA genotypes might affect episodic memory-related information processing in the human hippocampus.

Because the hippocampus is especially activated by associative episodic memory tasks $(21,22)$, we tested the impact of the $K I B R A$ genotype on hippocampal activations in a face-profession association task. During memory retrieval, noncarriers of the $\mathrm{T}$ allele showed significantly increased brain activations compared with those of $\mathrm{T}$ allele carriers in the medial temporal lobe [local maximum in the right hippocampus at coordinate position $(26,-12,-14), t=4.76, P<0.001$, coordinates according to the Montreal Neurological Institute] (Fig. 3). Noncarriers of the T allele also showed increased activations in the frontal cortex [local maxima in the right medial frontal gyrus (Brodmann area 8/9) at coordinate position $(30,42,42), t=4.24, P<$ 0.001 and in the left medial frontal gyrus (Brodmann area 6) at $(-24,10,56), t=4.38$, $P<0.001$ ] and in the parietal cortex [local maximum in the right inferior parietal lobule (Brodmann area 40) at coordinate position (50,-24,30), $t=3.97, P<0.001]$. The brain regions observed in these studies belong to a network important for episodic memory retrieval (23), which was also activated during

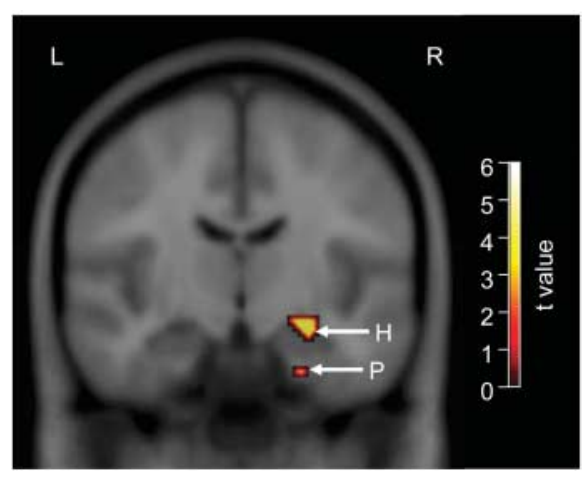

Fig. 3. Significant KIBRA allele-dependent differences in hippocampal activation as measured with fMRI. Activations are significantly increased in the hippocampus in noncarriers $(n=15)$ of the Tallele of SNP rs17070145 than in the hippocampus of T allele carriers $(n=15)$. Activations from all 30 individuals were overlaid on a coronal section of a T1-weighted magnetic resonance image of SPM2 and displayed in color-coded $t$ values. Threshold: $P<0.001$. H, hippocampus; $\mathrm{P}$, parahippocampal gyrus; $L$, left side of the brain; $R$, right side of the brain. our memory retrieval task (fig. S3), including the medial temporal lobe [local maximum in the right hippocampus at coordinate position $(32,-8,-24), t=4.27, P<0.001]$. There was no additional increased brain activation in noncarriers of the $\mathrm{T}$ allele in this episodic memory task. Furthermore, in a working memory task noncarriers of the $\mathrm{T}$ allele failed to show any increased retrieval-related brain activation compared with that of $\mathrm{T}$ allele carriers, indicating that the activations in non-carriers were specific to episodic memory retrieval.

As expected based on the matching for 5-min delayed recall performance, there were also no allele-dependent differences in retrieval performance of the fMRI task $(P=$ 0.5 ). Our findings therefore suggest that noncarriers of the $\mathrm{T}$ allele need more activation in these memory retrieval-related brain regions to reach the same level of retrieval performance as $\mathrm{T}$ allele carriers. There was no significantly increased task-related cortical activation in the $\mathrm{T}$ allele group as compared to noncarriers of the $\mathrm{T}$ allele. No allele-dependent differences in brain activations during encoding were found, suggesting that the genotype did not affect episodic memory at this early stage of memory formation. Consistent with this observation, there were no allele-dependent differences in tasks of early memory formation in the Swiss cohort 1 (i.e., immediate recall, Table 1 ) or in the U.S. cohort (i.e., AVLT learning trial one, $P>0.05$ ). Automated voxel-based algorithms [Statistical Parametric Mapping Software package (SPM2)] (24) and manual volume measurements failed to reveal significant allele-dependent differences in volumes of the hippocampus or the parahippocampal gyrus, or in white and gray matter volumes, suggesting that functional imaging results were not biased by morphological differences. Furthermore, there were no significant correlations between memory measures and any of the brain volumes.

Taken together, we present evidence from independent experiments suggesting a role for KIBRA in human memory. KIBRA was recently identified in a yeast two-hybrid screen as the binding partner for the human isoform of dendrin, a putative modulator of synaptic plasticity (17). A truncated form, which was expressed in the hippocampus, lacks the first 223 amino acids and contains a C2-like domain, a glutamic acid-rich stretch, and a protein kinase $\mathrm{C}$ (PKC) $\zeta$-interacting domain (25). PKC $\zeta$ is involved in memory formation and in the consolidation of longterm potentiation $(26,27)$. The C2-like domain of KIBRA is similar to the $\mathrm{C} 2$ domain of synaptotagmin, which is believed to function as the main $\mathrm{Ca}^{2+}$ sensor in synaptic vesicle exocytosis $(17,28)$. The memoryassociated KIBRA haplotype block and SNPs we describe map within the truncated KIBRA, which contains both the $\mathrm{C} 2$-like and the $\mathrm{PKC}$ interacting domains.

\section{References and Notes}

1. G. E. McClearn et al., Science 276, 1560 (1997).

2. D. J. de Quervain et al., Nat. Neurosci. 6, 1141 (2003).

3. M. F. Egan et al., Cell 112, 257 (2003).

4. A. Papassotiropoulos et al., Hum. Mol. Genet. 14, 2241 (2005).

5. D. J. de Quervain, A. Papassotiropoulos, Proc. Natl. Acad. Sci. U.S.A. 103, 4270 (2006)

6. N. J. Schork, Adv. Genet. 42, 299 (2001)

7. J. R. Kelsoe, Int. Rev. Psychiatry 16, 294 (2004).

8. D. W. Craig, D. A. Stephan, Expert Rev. Mol. Diagn. 5, 159 (2005)

9. R. J. Klein et al., Science 308, 385 (2005).

10. Materials and methods are available as supporting material on Science Online.

11. M. L. Freedman et al., Nat. Genet. 36, 388 (2004).

12. J. K. Pritchard, N. A. Rosenberg, Am. J. Hum. Genet. 65, 220 (1999)

13. L. R. Squire, P. Alvarez, Curr. Opin. Neurobiol. 5, 169 (1995).

14. R. J. Caselli et al., Neurology 62, 1990 (2004).

15. S. J. Rosenberg, J. J. Ryan, A. Prifitera, J. Clin. Psychol. 40, 785 (1984)

16. D. G. Clayton et al., Nat. Genet. 37, 1243 (2005).

17. J. Kremerskothen et al., Biochem. Biophys. Res. Commun. 300, 862 (2003)

18. S. Y. Bookheimer et al., N. Engl. J. Med. 343, 450 (2000).

19. B. Milner, Clin. Neurosurg. 19, 421 (1972).

20. D. L. Schacter, E. Tulving, Memory Systems (MIT Press, Cambridge, 1994).

21. N. Degonda et al., Neuron 46, 505 (2005).

22. K. Henke, B. Weber, S. Kneifel, H. G. Wieser, A. Buck, Proc. Natl. Acad. Sci. U.S.A. 96, 5884 (1999).

23. R. Cabeza, L. Nyberg, J. Cognit. Neurosci. 12, 1 (2000)

24. J. Ashburner, K. J. Friston, Neuroimage 11, 805 (2000).

25. K. Buther, C. Plaas, A. Barnekow, J. Kremerskothen, Biochem. Biophys. Res. Commun. 317, 703 (2004).

26. E. A. Drier et al., Nat. Neurosci. 5, 316 (2002).

27. T. C. Sacktor et al., Proc. Natl. Acad. Sci. U.S.A. 90, 8342 (1993).

28. J. Ubach, X. Zhang, X. Shao, T. C. Sudhof, J. Rizo, EMBO J. 17, 3921 (1998)

29. Haploview 3.2 (www.broad.mit.edu/mpg/haploview).

30. Allen Institute for Brain Science, Allen Brain Atlas (www.brain-map.org)

31. Supported by grants from the Swiss National Science Foundation (PP00B-68859 to A.P., PP00B-106708 to D.Q., and 3100-067114 to K.H.), the Helmut-Horten Stiftung (D.Q. and A.P), the Hermann-Klaus Stiftung (D.Q.), the Emily Dorothy Lageman-EMDO Stiftung (D.Q.), the Olga Mayenfisch Stiftung (A.P.), the Baugarten Stiftung (A.P.), the National Institute of Mental Health (R01MH057899 to E.M.R), the National Institute on Aging (P3OAG19610 to E.M.R.), the NIH Neuroscience Blueprint (U24NS051872 to D.A.S.), the Bisgrove charitable donation (D.A.S and D.W.C.), the State of Arizona (D.A.S and E.M.R), and the Endgame U01 grant U01HL086528-01 (D.A.S.). We gratefully acknowledge the Allen Brain Institute for the use of their elegant murine in situ resource. We thank J. Trent, J. Lemna, and D. Locke for assistance.

\section{Supporting Online Material}

www.sciencemag.org/cgi/content/full/314/5798/[PAGE]/DC1 Materials and Methods

Figs. $\mathrm{S} 1$ to $\mathrm{S5}$

Table S1

References

11 May 2006; accepted 20 September 2006 10.1126/science. 1129837 